Revista de Ciencias Sociales - Número 67 (2015) - Páginas 151-173

Las políticas de la diferencia en el Estado democrático de Derecho

\title{
LAS POLÍTICAS DE LA DIFERENCIA EN EL ESTADO DEMOCRÁTICO DE DERECHO*
}

\author{
THE POLICIES OF THE DIFFERENCE IN \\ THE DEMOCRATIC RULE OF LAW
}

\author{
LUIS VILLAVICENCIO MIRANDA* \\ Universidad de Valparaíso (Chile) \\ luis.villavicencio@uv.cl \\ NICOLE SELAMÉ GLENA** \\ Universidad de Valparaíso (Chile) \\ nselameg@gmail.com
}

\section{Resumen}

Este artículo examina si las tesis liberales igualitarias y los postulados multiculturalistas son compatibles. En primer lugar, se precisan algunos de

* $\quad$ Este trabajo forma parte del proyecto FONDECY'T N 1150094 titulado "Las mujeres indígenas como una minoría dentro de las minorías. Un caso difícil para la teoría y el derecho" cuyo investigador responsable es Luis Villavicencio Miranda. Nicole Selamé agradece a CONICYT el financiamiento otorgado a través del programa PAI (Concurso Nacional de Apoyo al Retorno de Investigadoras desde el Extranjero) $\mathrm{N}^{\circ} 82140027$. El presente artículo es una versión revisada de un trabajo de los mismos autores: "Liberalismo, multiculturalismo y Estado de bienestar". En: Ideas y Valores. Revista Colombiana de Filosofía, Universidad Nacional de Colombia, Volumen 60, No 146, 2011. Págs. 111-140.

* Profesor titular del departamento de Introducción al Derecho y Filosofía del Derecho. Doctor en Derecho por la Universidad Autónoma de Madrid. Dirección postal: Errázuriz 2120, Valparaíso. Artículo recibido el 19 de julio de 2015 y aceptado el 6 de noviembre de 2015 .

\footnotetext{
** Profesora del departamento de Introducción al Derecho y Filosofía del Derecho e investigadora proyecto CONICYT/ PAI. Doctora en Derecho por la Universidad Pompeu Fabra (Barcelona).
}

Revista de Ciencias Sociales - Número 67 (2015) - Universidad de Valparáíso - ISSN 0716-7725-Valparaíso, Chile 
los términos centrales que del debate. Luego, indaga si la dicotomía entre liberalismo y multiculturalismo admite alguna conciliación en orden a defender la idea de que una concepción del liberalismo igualitario que malinterprete la diversidad cultural puede constituirse en una forma de opresión que, más que fortalecer el Estado democrático de Derecho, lo debilite significativamente. Y, para terminar, se señalan algunas conclusiones encaminadas a mostrar la compatibilidad entre el multiculturalismo y el Estado de Bienestar.

\title{
Palabras clave
}

Liberalismo igualitario, multiculturalismo, diversidad cultural, Estado de Derecho, opresión.

\begin{abstract}
This article examines whether the thesis of egalitarian liberals are consistent with the multiculturalists thesis. Foremost, it explains some of the key terms of the discussion. Subsequently, it inquires if the dichotomy between liberalism and multiculturalism admits some reconciliation in order to defend the idea that a conception of egalitarian liberalism that misunderstands cultural diversity can become a form of oppression that, rather than strengthening the Democratic Rule of Law, it considerably weakened. Finally, it proposes a few conclusions aimed to support the compatibility between of multiculturalism and the Welfare State.
\end{abstract}

\section{Keywords}

Egalitarian liberalism, multiculturalism, cultural diversity, Rule of Law, oppression.

\section{Introducción}

Tradicionalmente aquellos que defendemos el Estado democrático de Derecho o el Estado de Bienestar intentamos justificar un conjunto de políticas públicas que buscan proveer ciertos derechos de manera universal con cargo a rentas generales —en áreas como la educación, la salud y pensiones — para cumplir el viejo anhelo liberal igualitario de conciliar los dos valores que constituyen a las democracias occidentales modernas, esto es, la libertad y la igualdad, sin que ello suponga equipararnos en la miseria como sucede (o sucedió) con los

Facultad de Derecho y Ciencias Sociales - Universidad de Valparaíso - Chile 
socialismos reales, ni nos haga esclavos del azar que nos asigna caprichosamente talentos, entorno familiar y social en medio de un capitalismo desatado que profundiza irremediablemente esas desigualdades de cuna.

Una política como la que propugna el Estado de Bienestar requeriría un ideal de ciudadanía fuertemente arraigado en valores republicanos comunes que alimenten la cohesión social indispensable para que aquellos que ganan más contribuyan a mejorar las oportunidades de los menos aventajados. Sin un grado de homogeneidad vigoroso pareciera que la estabilidad y los fines del Estado de Bienestar se encontrarían siempre amenazados.

Por esa razón, para el liberalismo igualitario las políticas multiculturalistas no serían una vía correcta para terminar con las desigualdades. La razón fundamental es que no consideran como referente las desventajas de las personas en cuanto a oportunidades o recursos, sino la pertenencia a un determinado grupo. Al "culturizarse" las identidades de grupo se dejan de lado los problemas comunes como la pobreza, el desempleo, la deficiencia educacional, la desnutrición, la precariedad habitacional, etc. Así, por ejemplo, Barry —el autor liberal igualitario que se ha ocupado expresamente de responder de manera sistemática al multiculturalismo en su obra Culture and Equalityplantea que frente al hecho irrefutable de la diversidad, la estrategia correcta es la privatización de los asuntos identitarios como la mejor forma de garantizar la igualdad de oportunidades. En este sentido, Barry considera que es positivo que el liberalismo sea "ciego a la diferencia", ya que los liberales no buscan erradicar las formas de vida tradicionales ni reclamar contra la mantención de las culturas ancestrales, sino simplemente oponerse a la coerción respecto de los que no comparten estos objetivos ${ }^{1}$. No están a favor ni en contra de la asimilación, sino que simplemente creen que la cantidad justa de ella es la que se da en un marco de instituciones equitativas ${ }^{2}$.

1. BARRY, Brian: Culture and Equality: An Egalitarian Critique of Multiculturalism. Harvard University Press, Cambridge, 2002. Pág. 66.

2. Para una presentación crítica del pensamiento de Barry, puede verse VILLAVICENCIO MIRANDA, Luis: "Privatizando la diferencia: el liberalismo igualitario y el pluralismo cultural”. En: Revista de Derecho (Valdivia), Volumen XXIII, $\mathrm{N}^{\circ}$ 1, 2010. Págs. 37-57.

Revista de Ciencias Sociales - Número 67 (2015) - Universidad de Valparáíso - ISSN 0716-7725-Valparaíso, Chile 
Los multiculturalistas, por su parte, señalan que este modelo de ciudadanía igualitaria, al comprender derechos universales, se olvida de las diferencias de género, raza o clase y, por lo tanto, no es adecuado en el contexto de un mundo culturalmente diverso. Lo que sostienen los multiculturalistas es que la diferencia no debe ser asimilada o excluida, y para evitarlo deben existir derechos especiales por grupos otorgados en razón de las características culturales que los distingan. Luego, la justicia social no debe definirse echando mano, exclusivamente, a reglas ciegas a la diferencia, sino recurriendo también al trato diferenciado como una especificación del principio de igualdad, entendido de una forma más inclusiva, que permite a la mayoría de la sociedad mostrarse más proclive a conceder determinadas excepciones al trato igualitario respetando así activamente la diferencia y la diversidad cultural.

En las palabras que siguen intentaremos reflexionar en torno a la cuestión de si las tesis liberales igualitarias y los postulados multiculturalistas realmente son irreconciliables entre sí. En particular, revisaremos si las políticas de la diferencia, que se alientan desde las filas multiculturalistas, son compatibles o, por el contrario, se oponen a las políticas de redistribución que caracterizan al Estado de Bienestar que propugna el liberalismo igualitario. Para ello, en primer lugar, precisaremos algunos de los términos centrales que del debate, los que constituyen los presupuestos del planteamiento, sin los cuales no se comprendería adecuadamente la conclusión. En segundo lugar, indagaremos si la dicotomía entre liberalismo y multiculturalismo admite alguna conciliación que permita defender la idea de que una concepción del liberalismo igualitario que malinterprete la diversidad cultural puede constituirse en una forma de opresión que, más que fortalecer el Estado de Bienestar, lo debilite significativamente. Y, para terminar, apuntaremos algunas conclusiones encaminadas a mostrar la compatibilidad entre el multiculturalismo y el Estado de Bienestar.

\section{Evitando equívocos}

Comenzaremos explicitando cómo entenderemos algunos conceptos claves. En primer lugar, nos referiremos a la distinción entre

Facultad de Derecho y Ciencias Sociales - Universidad de Valparaíso - Chile 
las políticas de redistribución y las políticas de la diferencia o reconocimiento. Apoyándonos en Kymlicka ${ }^{3}$, existen dos grandes formas de criticar el modelo tradicional de ciudadanía como derechos identificado con el liberalismo igualitario: por un lado, aquella perspectiva — llamada republicanismo — que demanda la necesidad de poner una mayor atención en las virtudes cívicas y la participación política activa; y, por otro, el punto de vista multicultural, que enfatiza la exigencia de al menos complementar el enfoque liberal dando un mayor énfasis a los derechos de grupo. Obviamente, para hacer la diferenciación que nos interesa nos concentraremos en la segunda perspectiva, la crítica multicultural al programa liberal.

Aclarado ese primer punto, nos interesa ahora avanzar en una segunda dirección: en qué sentido se conectan (y en qué sentido no) las políticas de la diferencia con la idea de la identidad social o comunitaria y como ello influye en el modo en que comprendemos y diferenciamos esas políticas de las de redistribución. Parekh considera indispensable superar la visión unidimensional de la identidad humana, propia del liberalismo igualitario, que considera a los seres humanos como agentes morales anteriores a sus fines por una concepción de la identidad humana tridimensional articulada por tres componentes inseparables e interconectados: la identidad personal, la identidad social o comunitaria, y la identidad humana global o universal ${ }^{4}$. La crítica multicultural se nutre de esa visión. La idea subyacente es que las sociedades se caracterizan por su amplia diversidad y su pluralismo cultural. Antes esa diversidad se aplastaba bajo el modelo del ciudadano "normal" (hombre no discapacitado, propietario, blanco y heterosexual), y quien se desviara del modelo era excluido, marginado, silenciado o asimilado. En la actualidad aquellos grupos que se sitúan en los márgenes o fuera de la concepción tradicional de ciudadanía, se vinculan con sus fines y su identidad social de manera no-electiva y, por lo mismo,

3. KYMLICKA, Will: Contemporary Political Philosophy: An Introduction (2 ${ }^{\mathrm{a}} \mathrm{ed}$ ). Oxford University Press, Oxford, 2002. Págs. 327-370.

4. PAREKH, Bhikhu: A New Politics of Identity. Political principles for an Interdependent World. Palgrave MacMillan, Basingstoke, 2008. Págs. 9-30.

Revista de Ciencias Sociales - Número 67 (2015) - Universidad de Valparáíso - ISSN 0716-7725-Valparaíso, Chile 
no basta con que la organización política asegure un marco igualitario donde las personas escojan sus fines y vínculos, sino que debe dárseles algún grado de protección pues sus horizontes de sentido se encuentran amenazados por la cultura mayoritaria ${ }^{5}$. Demandan una concepción de la ciudadanía más inclusiva, que reconozca sus identidades y dé cabida a sus diferencias.

Ahora bien, esa integración que reclaman las minorías culturales no es individual, sino grupal. Para ellos, la concepción tradicional de la ciudadanía fomenta la marginación o estigmatización de grupos que escapan del estereotipo artificial en el que se funda la ilusión del Estado nacional. Los derechos ideados para el "ciudadano normal" no se acomodan a las necesidades de esos grupos que reclaman una ciudadanía diferenciada que exige que las personas no sean integradas sólo como individuos, sino también a través de su grupo aglutinado en torno a alguna visión identitaria más o menos comprehensiva. Estas comunidades demandan formas específicas de ciudadanía ya sea porque rechazan la idea de una cultura nacional común (como sería el caso de los pueblos indígenas) o ya sea porque creen que es la mejor forma de integrarse (como sería el caso de colectivos homosexuales y algunas minorías religiosas).

Pero, ¿qué explica tales reivindicaciones? Se puede sostener que al interior de los Estados modernos conviven dos tipos principales de jerarquías: la económica y la asociada al estatus. La posición que una persona ocupa en la jerarquía económica está determinada por su relación con el mercado y los medios de producción. La lucha contra las iniquidades inherentes a esta jerarquía genera las "políticas de redistribución". La jerarquía del estatus se refleja en una historia de reglas discriminatorias contra grupos de menor categoría, y su invisibilidad o carácter estereotipado. La lucha contra estas jerarquías genera las "políticas de reconocimiento o de la diferencia". A pesar de

5. VILLAVICENCIO MIRANDA, Luis: "La concepción política de la persona y las visiones constitutivas del bien”. En: Ideas y Valores. Revista Colombiana de Filosofía, Universidad Nacional de Colombia, No 135, 2007. Págs. 29-49.

6. FRASER, Nancy: "Rethinking Recognition”. En: New Left Review, MayoJunio, No 3, 2000. Págs. 107-120; DELANTY, Gerard: Community (2 ed.). Routledge, Abingdon, 2010. Pág. 59; y MODOOD, Tariq: Multiculturalism. A Civic Idea. Polity, Cambridge, 2007. Págs. 68-70.

Facultad de Derecho y Ciencias Sociales - Universidad de Valparaíso - Chile 
que podamos distinguir las políticas de redistribución y reconocimiento para fines analíticos, lo cierto es que en el mundo real aparecen a menudo superpuestas (v.gr. piénsese en los mapuches que son al mismo tiempo una minoría nacional, étnica y social) al punto que para el liberalismo y para el marxismo la jerarquía del estatus es puramente accesoria. Con todo, la evidencia sugiere que la jerarquía del estatus no es reducible a la jerarquía económica. Como prueba de lo anterior, podemos señalar casos de grupos económicamente bien posicionados, pero culturalmente estigmatizados, como los gays, ciertos inmigrantes y ciertos grupos religiosos; y, a la inversa, casos de grupos que gozan de una posición privilegiada en la jerarquía del estatus, pero que se encuentran (o se encontraban) en desventaja económica, como la clase trabajadora masculina en la mayoría de las democracias occidentales.

Así, podemos señalar que no hay una correlación simple entre ambas jerarquías. Esto explica por qué la estrategia de una ciudadanía común funcionó para la clase trabajadora masculina, pero no satisfizo a otros grupos, las mujeres sin ir más lejos, quienes necesitan además un ataque a las jerarquías sustentadas en el estatus pues constituyen un grupo estructuralmente oprimido que, subyugadas por un contrato sexual suscrito bajo la amenaza patriarcal, transfieren gratuitamente su fuerza de trabajo a los hombres haciéndose cargo casi exclusivamente de las labores domésticas, de crianza y de cuidado7.

Nos detenemos, a continuación, en una tercera precisión. Defender la conexión entre la idea de identidad y las políticas de la diferencia o reconocimiento no implica confundirlas con las políticas de la identidad esencialistas. Como postula Judith Squires ${ }^{8}$, es un error común confundir una política de la identidad esencialista con una política de la diversidad constructivista. Sin embargo, ambas son bastante distintas: la primera cree en una esencia verdadera e irreductible

7. Cfr. YOUNG, Iris Marion: "A Multicultural Continuum: A Critique of Will Kymlicka's Ethnic-Nation Dichotomy". En: Constellations, Volumen 4, No 1 , 1997. Págs. 48-53; y de la misma autora: La justicia y la política de la diferencia. Cátedra, Madrid, 2000. (Trad. Silvina Álvarez). Págs. 89-93.

8. SQUIRES, Judith: "Culture, Equality and Diversity". En: Kelly, Paul (ed.): Multiculturalism Reconsidered. Polity Press, Cambridge, 2002. Págs. 114-132.

Revista de Ciencias Sociales - Número 67 (2015) - Universidad de Valparáíso - ISSN 0716-7725-Valparáiso, Chile 
que no cambia, mientras que la segunda considera que la esencia es una construcción histórica y no algo dado naturalmente. Ambas versiones sostienen proyectos políticos diferentes, uno esencialista - estableciendo para sus miembros los derechos, el reconocimiento y los privilegios que los grupos dominantes buscan monopolizar-, y el otro constructivista - buscando la deconstrucción de los supuestos y demandas que dicen relación con que la identidad tiene una esencia intrínseca, y la comprensión de que las identidades responden siempre a construcciones sociales, contextual y necesariamente constituidas a través de la exclusión-; el problema es que en la práctica política es difícil desentrañarlas. De todos modos, los que defienden la política de la diferencia buscan distanciarse lo más posible del esencialismo, mientras que los defensores de la política de la identidad lo invocan de manera consciente. Squires cree que la distinción entre política de la diversidad y política de la identidad es importante, porque "permite que aceptemos el rechazo de Barry [y del liberalismo igualitario en general] de las políticas de la identidad, defendiendo al mismo tiempo las demandas de reconocimiento de las diferencias que emergen de la perspectiva de las políticas de la diversidad"9.

Parekh, por su parte, lo plantea de este modo: muchos multiculturalistas, incluyéndose, no creen que las culturas sean entes cerrados que no estén sujetos a revisión y alteración de sus contenidos. Discrepan fuertemente de la tesis, defendida por el liberalismo y en particular por Barry, de que la cultura tiene una importancia marginal y que la identidad personal sea suficiente para explicar el comportamiento humano. Como insiste Parekh, la naturaleza humana nunca se presenta en su forma natural y está ineludiblemente moldeada y estructurada por la cultura. Esto último destaca ciertos aspectos de la naturaleza humana en vez de otros, les da cierta orientación y desarrolla su personalidad de manera particular; es en este importante sentido en que los seres humanos están insertos culturalmente y experimentan sus vidas dentro de un marco cultural ${ }^{10}$.

9. Ibíd. Pág. 116.

10. PAREKH, Bhikhu: Rethinking Multiculturalism: Cultural Diversity and Political Theory (2 ed.). Palgrave MacMillan, Londres, 2006. Págs. 349-350.

Facultad de Derecho y Ciencias Sociales - Universidad de Valparaíso - Chile 
Pero lo anterior no significa que las personas queden "fijas" en su cultura, sino solamente que no se puede atender a sus necesidades como si vivieran en un vacío. Los seres humanos pueden revisar sus culturas y modificarlas, y precisamente por eso es que es importante el diálogo con las otras. "Es justamente porque cada cultura tiene sus limitaciones que los multiculturalistas consideran a la diversidad cultural como un bien moral vital; y argumentan que los seres humanos necesitan no sólo una rica y consistente cultura en donde desarrollarse, sino también acceso a las demás. [...] A través de un diálogo comprensivo con otras culturas, comenzamos a apreciar las fortalezas y límites de la nuestra, tomamos conciencia respecto a qué es aquello que la distingue, así como también aquello que comparte con las demás, y tenemos la oportunidad de enriquecernos a través de un préstamo juicioso de sus características más atractivas" ${ }^{11}$.

Cerramos este apartado con una cuarta precisión: debe distinguirse cuidadosamente el multiculturalismo como ideología y el multiculturalismo como política. Como señala David Miller, "multiculturalismo" es un término vago que a veces se utiliza en un sentido puramente descriptivo para referirse al hecho de la diversidad. Pero lo cierto es que este uso puede llevar a confusión, por lo que es mejor hablar a este respecto de "diversidad cultural", "pluralismo cultural" o "diferencia cultural". Sin embargo, existen también otros significados del término usados comúnmente, que son los que, por ejemplo, Barry desconoce. "Multiculturalismo" puede ser utilizado en un sentido normativo, en referencia a una ideología que otorga valor positivo a la diversidad cultural, busca el igual reconocimiento de los grupos culturales y llama al Estado a apoyar estos grupos en variadas formas. Esta ideología puede manifestarse heterogéneamente y en distintos grados; así, podrá ser más fuerte o más débil en atención al nivel de compromiso con la diversidad y la radicalidad de sus demandas. Pero el término también da cuenta del conjunto de políticas que se crean para ayudar a las minorías culturales material o simbólicamente, lo que es algo distinto ${ }^{12}$. Siguiendo el análisis de Miller, es relevante

11. Ibíd. Pág. 350.

12. MILLER, David: "Multiculturalism and the Welfare State: Theoretical Reflections”. En: Kymlicka, Will y Banting, Keith (ed.): Multiculturalism and the Welfare State: Recognition and Redistribution in Contemporary Democracies. Oxford University Press, Oxford, 2006. Págs. 323-338.

Revista de Ciencias Sociales - Número 67 (2015) - Universidad de Valparáíso - ISSN 0716-7725-Valparaíso, Chile 
distinguir el multiculturalismo como ideología del multiculturalismo como política pública por dos motivos: a) un país que ideológicamente rechace el multiculturalismo puede mantener políticas para promoverlo en su agenda pública, o viceversa; y b) la mayoría de los críticos del multiculturalismo no está en contra de la acción afirmativa, exenciones para las minorías de las normas generales o educación bilingüe, sino que les preocupa que se genere una cultura política que dé más valor a estos asuntos que a los problemas comunes relativos a la redistribución de recursos escasos. Y vistas así las cosas, no es temerario aventurar que en torno a esta problemática tenemos, a pesar de las apariencias, más coincidencias que desacuerdos. Pero no nos adelantemos, revisemos la argumentación paso a paso.

\section{El liberalismo igualitario es compatible con el multiculturalismo}

Barry piensa que la mejor forma de lidiar con las iniquidades es sustraer al Estado de los problemas que genera la diversidad, "privatizando la diferencia". En la medida que el Estado se mantenga neutral, podrán florecer libremente los distintos modos de vida que quepan dentro del marco garantista establecido por los principios liberales y las fuerzas políticas podrán orientarse a la resolución de los problemas "verdaderamente importantes": los que dicen relación con la redistribución de recursos escasos. Una vez resueltos éstos, las demandas de reconocimiento serán mínimas, pues las personas estarán en condiciones de autogestionar su propio modo de vida y desarrollarse en él, sin vulnerar derechos fundamentales. Creo, sin embargo, que no todos los problemas pueden reconducirse a la redistribución de los recursos. El liberalismo igualitario de Barry, antes que una respuesta, puede ser una forma de opresión y las políticas multiculturalistas, por su parte, pueden fortalecer el Estado de Bienestar, pues no se oponen a las políticas de redistribución, sino que las complementan. Las preguntas que debemos formulamos son, entonces, si acaso un liberal igualitario puede ser multiculturalista, y si son compatibles las políticas multiculturalistas con el Estado de Bienestar.

Una primera aproximación al problema parece arrojar respuestas claramente negativas. Sin embargo, a pesar de esta aparente oposición,

Facultad de Derecho y Ciencias Sociales - Universidad de Valparaíso - Chile 
creemos que un liberal igualitario sí puede ser multiculturalista; claro está, siempre y cuando no piense como Barry, quien incluso arriesga sus credenciales de igualitario. Éste considera que multiculturalismo y liberalismo son doctrinas incompatibles, y que cualquier intento por reconciliarlos termina por menoscabar al primero, pues el multiculturalismo rechaza las tres premisas básicas del liberalismo igualitario, esto es, que existe una naturaleza humana universal de la que derivan intereses comunes, que no existen instituciones ni prácticas que se autovaliden y que el Estado debe tratar a todos los individuos de la misma forma ${ }^{13}$. Parekh, por el contrario, cree que el multiculturalismo, o algunas de sus vertientes, sí son compatibles con la teoría liberal. Resiste el esencialismo doctrinal y señala que no hay que asumir que existe una sola y verdadera forma de ser liberal. Afirma que la "consistencia" que busca darle Barry al liberalismo se transforma en una especie de fundamentalismo intolerante susceptible de las mismas críticas que él realiza a las políticas de la diferencia. Parekh señala que es necesario romper la "obsesión con la identidad doctrinal y retener nuestra libertad para explorar y experimentar. En vez de hablar de liberalismo [...] debemos desfragmentarlo en los principios liberales o valores, para permanecer libres de aceptar algunos, pero no todos, y combinarlos con aquéllos que provienen de otras fuentes. Los valores liberales se encuentran insertos y se nutren de una cultura particular o forma de comprender y organizar la vida humana. Es por esta razón que acogen ciertas preocupaciones sociales y proyectos, llevan la cultura consigo adonde sea que vayan y toman diferentes formas dependiendo de cómo ello los transforma y a su vez éstos transforman a la cultura local. Tal como la visión liberal de la vida, otras formas de vida [...] contienen visiones valiosas de la inmensa complejidad y diversidad de ésta y encarnan valores admirables. Debemos llevar estos entendimientos y valores a un diálogo creativo y crítico con la visión liberal de la vida, y abrir las posibilidades de nuevos sistemas de pensamiento más relajados respecto de la identidad, cruzar las fronteras doctrinarias con soltura y confianza, y resistir las rotulaciones" ${ }^{14}$.

13. PAREKH, Bhikhu: Rethinking Multiculturalism, ob. cit. Pág. 347.

14. Ibíd. Pág. 369.

Revista de Ciencias Sociales - Número 67 (2015) - Universidad de Valparáíso - ISSN 0716-7725-Valparaíso, Chile 
Susan Mendus aborda la pregunta desde otra perspectiva, oponiendo las posiciones de Barry y Parekh. Para ella, lo que ocurre es que ambos autores tratan de enfrentarse a la diversidad cultural desde el liberalismo igualitario, pero con concepciones de la justicia distintas. Por una parte, Barry cree que la justicia tiene que ver, sobre todo, con la igualdad de oportunidades, y que esta igualdad es un estado de cosas objetivo; por la otra, Parekh piensa que la oportunidad requiere de una cierta disposición subjetiva, arraigada culturalmente. Mendus considera que el hecho de que alguien tenga o no una oportunidad depende de los costos asociados a la actividad en cuestión, y cree que la disputa de si la oportunidad es o no subjetiva descansa, a su turno, en la disputa respecto del estatus de la religión y las creencias culturales. Así, la gran diferencia que separa el pensamiento de ambos autores es que Barry considera que la cultura es un asunto respecto del cual los agentes deciden, mientras que Parekh piensa que es algo que tiene que ver con la suerte o el azar. En una analogía simplista, podríamos decir que para Barry la cultura es similar a un gusto caro, y para Parekh a una discapacidad física.

De este modo, lo que plantea Mendus es que en principio un liberal igualitario podría ser multiculturalista, siempre y cuando considere que la religión y las costumbres caen en el lado de la suerte, y no en el de las opciones. El problema es que esto supone, evidentemente, que seamos capaces de trazar una línea preinstitucional y clara entre lo que corresponde a la suerte y lo que corresponde a nuestras decisiones voluntarias, lo que no es nada simple. De hecho, la gran crítica de Mendus a Barry es que al establecer esa distinción, en vez de (o junto con) atacar al multiculturalismo, socava las propias bases de su liberalismo igualitario, tornándolo opresivo. La cuestión crucial que debemos comprender es que la protesta igualitaria no debería reducirse simplemente a defender que es injusto negarse a compensar los efectos de la mala suerte, sino ir un paso más allá, esto es, remover la opresión social como causa de esas injusticias. Al establecer la línea divisoria suerte-decisión nuestro cuestionamiento central es si la religión y la cultura son elegidas o dadas, y perdemos de vista el problema realmente importante: si el compromiso religioso o cultural puede oponerse a nuestra capacidad para tener una vida plena, o si lo que entendemos

Facultad de Derecho y Ciencias Sociales - Universidad de Valparaíso - Chile 
por vida plena es en parte una función del compromiso cultural y religioso ${ }^{15}$.

Para Barry, la cultura y la religión pueden constituir un obstáculo para alcanzar una vida buena, y por eso propone un marco de instituciones liberales que, en un plano neutral, garantice el desarrollo de las distintas formas de vida con resguardo de ciertos valores (liberales) universales e inquebrantables. Como Barry cree que el núcleo de la vida buena está más allá de toda creencia o práctica religiosa o cultural, estima que lo más sensato es resguardarlo, aunque limite nuestro desarrollo identitario. Sin embargo, lo cierto es que nada asegura que esta protección liberal sea efectivamente neutral. Por el contrario, pensamos que al evitar que el Estado reconozca públicamente, fomente o apoye otras culturas, lo que hace es privilegiar la reproducción de la cultura dominante y desequilibrar el circuito de poder. Caney señala que una teoría de la justicia distributiva debe necesariamente operar sobre una visión de los intereses primarios de las personas, para determinar qué constituye un beneficio y qué una carga. Si pensamos que la tarea del Estado es distribuir diferentes oportunidades, usará como criterio de selección — para optar entre ellas- el que sirvan a intereses primarios valiosos. Pero, ¿̇qué intereses primarios defiende Barry y por qué razones? ${ }^{16}$. Sin muchas explicaciones ni fundamentos, Barry aboga exclusivamente por los valores liberales occidentales.

Shapiro sostiene que la meta de todo gobierno debe ser limitar la posibilidad de dominación, minimizando su interferencia en la consecución de los fines de las personas ${ }^{17}$. El modelo de Barry hace todo lo contrario, pues fortalece el poder de las mayorías sobre las minorías y respalda las atribuciones del Estado sobre la vida de las

15. MENDUS, Susan: "Choice, Chance and Multiculturalism”. En: Kelly, Paul (ed.): Multiculturalism Reconsidered. Polity Press, Cambridge, 2002. Págs. 31-44.

16. CANEY, Simon: "Exceptions and Cultural Diversity". En: Kelly, Paul (ed.): Multiculturalism Reconsidered. Polity Press, Cambridge, 2002. Págs. 90-98.

17. SHAPIRO, Ian: "Democratic Justice, Multicultural Recognition”. En: Kelly, Paul (ed.): Multiculturalism Reconsidered. Polity Press, Cambridge, 2002. Pág. 179.

Revista de Ciencias Sociales - Número 67 (2015) - Universidad de Valparáíso - ISSN 0716-7725-Valparaíso, Chile 
personas al establecer un único ideal de vida buena al que los demás deben adecuarse. En palabras de Parekh, busca que se cumpla el rol homogeneizante del Estado moderno, esperando que todos los ciudadanos se definan del mismo modo y se relacionen de igual manera entre sí y el Estado. El problema es que en una sociedad multiétnica y multinacional puede generar inestabilidad o secesión, y —según hemos señalado- convertirse en un instrumento de injusticia y opresión ${ }^{18}$.

Veamos, ahora, nuestro problema desde otra visión. Cuestionémonos si acaso las políticas multiculturalistas producen efectos negativos respecto de las políticas de redistribución. Para el liberalismo igualitario, la respuesta es obvia: puesto que cree que la política es un juego de suma cero, cualquier asunto que pase a formar parte activa de la agenda pública divertirá los esfuerzos que debieran destinarse a la redistribución. Pensamos, sin embargo, que además de no existir fundamento empírico para tal afirmación respecto de las políticas de reconocimiento ${ }^{19}$, es necesario previamente cuestionar el planteamiento mismo de la pregunta. ¿'Tiene sentido oponer dos presupuestos tan importantes para la justicia como la redistribución de los recursos escasos y el reconocimiento de las diferencias? ¿No forman ambos parte de la idea de dignidad humana? ¿Puede, en todo caso, disociarse uno de otro? Pareciera que el asunto es más complejo de lo que ha planteado Barry. El que la redistribución sea necesaria, no la hace suficiente. De modo que, en vez de plantear el tema en términos excluyentes, lo que debería hacerse es redefinir el debate sobre la justicia para lograr una teoría que integre tanto el reconocimiento como la redistribución.

Permítannos ahondar en este punto. Siguiendo a Kymlicka y Banting, el debate acerca del impacto que tienen las políticas multiculturalistas puede, en realidad, ser separado en dos distintos ${ }^{20}$.

18. PAREKH, Bhikhu: Rethinking Multiculturalism, ob. cit. Pág. 16.

19. Para un profundo desarrollo del tema, desde un punto de vista teórico y empírico, véase Kymlicka, Will y Banting, Keith (ed.): Multiculturalism and the Welfare State: Recognition and Redistribution in Contemporary Democracies. Oxford University Press, Oxford, 2006.

20. Ibíd. Pág. 49.

Facultad de Derecho y Ciencias Sociales - Universidad de Valparaíso - Chile 
Por una parte, hay quienes temen que la diversidad etno-lingüística o racial por sí misma debilite el Estado de Bienestar, al dificultar la generación de sentimientos de confianza y solidaridad entre los grupos. Y, por la otra, hay quienes creen que la adopción de políticas multiculturalistas para reconocer y acomodar grupos étnicos genera dinámicas políticas que inadvertidamente minan el Estado de Bienestar de las democracias occidentales. Ambos cuestionamientos suelen presentarse conectados y lo común es que quienes sostengan una de las hipótesis también suscriban la otra, a pesar de que no existan fundamentos empíricos ni teóricos para ello. Veamos por qué se incurre en ese error.

En primer lugar, nos encontraríamos con el efecto de desplazamiento o exclusión (crowding-out effect). Según la mayoría de los críticos liberales del multiculturalismo, incluyendo a Barry, las políticas de la diferencia hacen que se distraigan el dinero, la fuerza, el tiempo y los demás recursos que debieran destinarse a la redistribución, hacia las prácticas destinadas a fomentar el reconocimiento. Sin embargo, de acuerdo con lo planteado por Kymlicka y Banting, ello no es así. La afirmación de que el multiculturalismo quita fuerzas al Estado de Bienestar se fundamenta en la creencia — gratuita - de que existiría una masa de gente dispuesta a actuar en defensa de éste, pero que se ve distraída por el multiculturalismo. En el hecho esto no es efectivo; y baste para ello con mirar al ciudadano medio. Si la gente ha dejado de participar activamente o ha disminuido su actuación en pos de la redistribución, es mayoritariamente porque el Estado de bienestar mismo se encuentra en crisis. La pasividad de la izquierda no tiene que ver con el multiculturalismo, sino con sus propios fracasos. En este sentido, las políticas multiculturalistas tienden a significar más un avance en el agenciamiento político-ciudadano que un retroceso, y permiten que las personas puedan volver a mezclarse en política sintiendo que es posible hacer una diferencia. Desde este punto de vista, "el real desafío es que la gente se involucre en política [...]. Una vez que se encuentran involucrados, y tienen este sentido de eficiencia política, estarán abiertos a apoyar otras demandas progresistas también”"21.

21. Ibíd. Pág. 16.

Revista de Ciencias Sociales - Número 67 (2015) - Universidad de Valparáíso - ISSN 0716-7725-Valparaíso, Chile 
En segundo lugar, podemos identificar el supuesto efecto corrosivo (corroding effect) del multiculturalismo conforme al cual se sugiere que las políticas multiculturalistas debilitan la redistribución erosionando la confianza y solidaridad entre los ciudadanos. Según este punto de vista, el error del multiculturalismo está en realzar lo que diferencia a las personas en vez de lo que las hace iguales. De este modo, la gente que históricamente apoyaba el Estado de Bienestar, pues sentía que ayudaba a "alguien como él mismo", con una identidad común y un mismo sentido de pertenencia, deja de hacerlo, pues considera que ahora se trata de un "otro extraño". Frente a esta afirmación, Kymlicka y Banting insisten en que los críticos del multiculturalismo asumen que con anterioridad a la implementación de las políticas multiculturalistas existían altos niveles de solidaridad y confianza interétnica, y se olvidan que la historia de Occidente está marcada por políticas asimilacionistas y de exclusión, precisamente porque no existía dicha confianza y solidaridad. "Los grupos dominantes se sentían asustados frente a las minorías, y/o superiores a ellos, y/o simplemente indiferentes respecto de su bienestar, así que intentaban asimilarlas, excluirlas, explotarlas o quitarles su poder. Esto, a su turno, llevó a las minorías a desconfiar del grupo dominante"22. De este modo, podemos ver que las políticas multiculturalistas no son la causa original de la desconfianza, sino medidas que se toman a consecuencia de ella, de manera que los grupos mayoritarios no teman o desprecien a las minorías, y éstas últimas confíen en la sociedad. El reconocimiento de las injusticias cometidas actúa como confirmación del deber público de combatirlas en el futuro.

Por último, nuestros autores se refieren a una tercera línea de pensamiento que sugiere que el multiculturalismo produce un diagnóstico equivocado respecto de los problemas sociales (misdiagnosis effect). Consideran, como lo hace Barry por ejemplo, que esta corriente "culturiza" los problemas, esto es, que encuentra en la base de toda desventaja la falta de reconocimiento cultural, en circunstancias que la cultura no es ni la pregunta ni la respuesta al tema en cuestión. Los críticos señalan que, de este modo, las medidas que se toman no generan beneficios para los desaventajados, o lo hacen en una medida muy baja,

22. Ibíd. Pág. 17.

Facultad de Derecho y Ciencias Sociales - Universidad de Valparaíso - Chile 
pues se pierden de vista o se malentienden las causas reales de los problemas. Al centrarse únicamente en las diferencias étnicas y culturales, se dejan de lado los problemas económicos comunes. Lo principal de esta crítica no apunta simplemente a los recursos que se distraen (como en el efecto de desplazamiento o exclusión), sino a la distorsión en la comprensión de las causas de las iniquidades. Nos parece que esta crítica tendría pleno sentido si el multiculturalismo efectivamente tomara como única causa de los problemas la falta de reconocimiento cultural de las minorías. Pero eso es un error: el multiculturalismo no ignora otras causas ni minimiza su importancia, sino que simplemente agrega al debate público otra fuente de desigualdades. Kymlicka y Banting señalan una posible razón por la cual podría pensarse que considerar la cultura como raíz de injusticias anula otros factores como la clase y el género: si las personas tuvieran un sentido de justicia limitado, entonces, al dar relevancia a un determinado tipo de injusticia, necesariamente deberían desestimar otro. Pensamos, por el contrario, que lo más probable es que el sentido de justicia se vaya desarrollando y las personas incrementen su sensibilidad frente a diversas circunstancias, a medida que tomen conocimiento de ellas.

"Para evitar diagnósticos equivocados, lo importante es que la gente evite $[\ldots]$ presupuestos dogmáticos. Como la relevancia de ciertos tipos de desventajas difieren entre los distintos grupos, y a través del tiempo, es importante que la gente tenga la mente abierta [...] y esté dispuesta a considerar las demandas y evidencias como sean presentadas por los distintos grupos. [...] ¿A Alienta [el multiculturalismo] a la gente a asumir que las desigualdades culturales son el verdadero problema, sin examinar la evidencia de los casos particulares? ¿O, en vez de eso, generan las políticas multiculturalistas el espacio para un debate abierto sobre su relativa importancia?"'23. En definitiva, tanto en la teoría como en la práctica las políticas multiculturalistas han contribuido al debate. $\mathrm{Al}$ rechazar la teoría monocausal de la historia de las desigualdades, contravienen la idea de que las desventajas puedan reducirse a una sola injusticia y permiten una visión menos sesgada de los problemas

23. Ibíd. Pág. 20.

Revista de Ciencias Sociales - Número 67 (2015) - Universidad de Valparáiso - ISSN 0716-7725-Valparáís, Chile 
sociales. Creemos que ampliar el espectro de entendimiento no reduce las luchas sociales, sino que las fortalece.

\section{A modo de conclusión}

Terminamos con algunas ideas a modo de conclusión. Hemos sometido a crítica la tesis defendida por Barry de que una lectura correcta del liberalismo igualitario debería llevarnos, necesariamente, a rechazar las políticas de la diferencia por su supuesta incompatibilidad con las políticas de redistribución. Un ejemplo del propio autor resulta, a estas alturas, esclarecedor para comprender el alcance de su postura: el poder de veto sobre las políticas públicas que tienen ciertas minorías étnicas o nacionales se traduce, paradojalmente, en un bloqueo que perpetúa el statu quo. Dado que los que tienen ese derecho son, por lo general, los grupos desaventajados y oprimidos de la sociedad, tener la potestad de veto los habilita tan sólo para evitar los cambios perjudiciales para sus intereses. Una meta demasiado modesta y además costosa, sentencia Barry, para tanto alboroto multiculturalista. Costosa porque la tendencia endémica del multiculturalismo de asumir que los atributos culturales son los rasgos distintivos de todos los grupos, lleva al error de considerar que cualquier injusticia que enfrenta el grupo se relaciona con esos atributos, bloqueando las políticas de la redistribución en áreas tan relevantes como la educación, el trabajo digno y la salud. Al final de cuentas, el multiculturalismo haría que la persecución de su agenda haga más difícil alcanzar las políticas igualitarias, al menos, en dos modos: "como mínimo, desvía la oferta política lejos de las metas universalistas. Pero un más serio problema es que ese multiculturalismo puede fácilmente destruir las condiciones para articular una coalición transversal a favor de la nivelación de oportunidades y recursos" ${ }^{24}$.

Creemos que Barry yerra por varias razones. En primer lugar, la expresión "multiculturalismo" puede ser utilizada normativamente para aludir a una ideología que mira con buenos ojos la diversidad cultural buscando el igual reconocimiento de los grupos culturales por parte

24. BARRY, Brian: Culture and Equality: An Egalitarian Critique of Multiculturalism. Harvard University Press, Cambridge, 2002. Pág. 325.

Facultad de Derecho y Ciencias Sociales - Universidad de Valparaíso - Chile 
del Estado. En ese sentido, el multiculturalismo podrá ser más o menos intenso dependiendo de la radicalidad de sus demandas, pero lo decisivo es que, en sus formas más moderadas ${ }^{25}$, rechazaría un modelo esencialista de las identidades de grupo que permitiría desestimar las políticas de la identidad distinguiéndolas de las políticas de la diversidad, siendo éstas últimas perfectamente compatibles con las políticas de redistribución ${ }^{26}$. Por otra parte, el término "multiculturalismo" también se refiere al conjunto de políticas públicas que se institucionalizan con la finalidad de ayudar a las minorías culturales material o simbólicamente. Reparar en esta distinción es relevante pues puede suceder que un Estado rechace la ideología del multiculturalismo, pero mantenga políticas públicas que busquen beneficiar o igualar a dichas minorías ${ }^{27}$.

En segundo lugar, apoyándonos en la comparación que Mendus hace entre la postura de Barry y la de Parekh, podemos suscribir la idea de que un liberal igualitario podría ser multiculturalista siempre y cuando considere que la identidad cultural no es un asunto respecto del cual los agentes morales decidan voluntariamente, sino que tiene que ver con el modo en que el azar condiciona culturalmente la configuración de la identidad individual. El problema es que lo anterior presupone, obviamente, que podamos trazar una línea preinstitucional y precisa entre lo que es consecuencia de la suerte y lo que procede de

25. Pensamos, por ejemplo, en Parekh (Rethinking Multiculturalism, ob. cit. Págs. 367-372), quien — si desea ser consecuente— debería aplicar al propio multiculturalismo su tesis de que, junto a la visión liberal de la vida, existen otras concepciones que contienen visiones valorables de la inmensa complejidad y diversidad de la existencia encarnadas en valores, prima facie, estimables, las que deben abrirse a un diálogo creativo y a nuevos sistemas de pensamiento, cruzando las fronteras doctrinarias con soltura y confianza, resistiendo rotulaciones esencialistas. La cuestión clave es percatarse de que esa actitud es sólo compatible con lo que Baumeister llama multiculturalismo tenue (thin multiculturalism) en oposición a un multiculturalismo denso (thick multiculturalism) de carácter esencialista. Véase BAUSMEISTER, Andrea: Liberalism and the 'Politics of Difference'. Edinburgh University Press, 2000. Pág. 58.

26. SQUIRES, Judith: "Culture, Equality and Diversity", ob. cit.

27. MILLER, David: "Multiculturalism and the Welfare State", ob. cit.

Revista de Ciencias Sociales - Número 67 (2015) - Universidad de Valparaíso - ISSN 0716-7725-Valparaíso, Chile 
nuestras decisiones autónomas, lo que está muy lejos de ser algo pacífico. Pero, a mayor abundamiento, continúa Mendus, al establecer una línea divisoria suerte-decisión erigimos como pregunta central la cuestión de si la religión y la cultura son elegidas o dadas, y perdemos de vista el problema realmente importante: si el compromiso religioso o cultural pueden obstaculizar nuestra capacidad para tener una vida plena. $\mathrm{Al}$ enfatizar la dicotomía suerte-decisión, los igualitaristas — liberales o multiculturalistas - se esfuerzan exclusivamente en contraatacar los efectos de la suerte omitiendo la remoción de la opresión social. Por eso mismo, las políticas multiculturalistas - entendidas como aquéllas que van más allá de los problemas de redistribución — pueden fortalecer el Estado de Bienestar pues no se oponen a las políticas de redistribución, sino que las complementan.

En tercer lugar, como plantea Kymlicka ${ }^{28}$, si bien es posible distinguir conceptualmente las políticas de la diferencia de las de redistribución, la verdad es que en la vida real aparecen mezcladas y suele ser difícil separarlas. Pero más importante todavía es que, como nos recuerda Parekh, no "[E]s difícil imaginar una sociedad en la cual las desigualdades económicas y otras sean reducidas drásticamente o incluso eliminadas, pero que tengan una visión degradante de mujeres, minorías étnicas, culturales y religiosas, homosexuales y otros. Después de todo, la igualdad económica no genera por sí misma respeto por la diversidad (...) Una sociedad igualitaria podría insistir en una sola y correcta forma de llevar una vida buena. Sus miembros disidentes gozan de igualdad social y económica, pero no de la igualdad para definir y afirmar su identidad. Están destinados a sentirse oprimidos en el sentido en que se les deniega el respeto público igualitario y la libertad de autodeterminación (...) La injusticia no sólo se produce cuando los individuos son explotados, manipulados o cuando se les niegan las condiciones materiales básicas para una vida buena; sino también cuando se les niega la oportunidad de hablar en sus propias voces, modelar y expresar libremente sus identidades (...) La opresión y la iniquidad pueden tomar muchas formas, la económica es sólo una de ellas. Aquellos que se avocan a la redistribución se concentran en una

28. KYMLICKA, Will: Contemporary Political Philosophy, ob. cit. Págs. 327-376.

Facultad de Derecho y Ciencias Sociales - Universidad de Valparaíso - Chile 
de ellas, aquellos que se enfocan en el reconocimiento, en otras"29. De este modo, nos parece que el camino a seguir no debe plantearse en los términos de escoger entre una u otra política, sino redefinir las políticas públicas de modo tal que armonicen el paradigma redistributivo con el paradigma del reconocimiento.

Para finalizar, volvemos al inicio: ¿es efectivo que las políticas multiculturalistas debilitan la redistribución de recursos erosionando la confianza y la solidaridad entre los ciudadanos? La única respuesta plausible es negativa. Los críticos del multiculturalismo olvidan que mucho antes de la implementación de políticas de la diferencia existían en los Estados modernos altos niveles de asimilación y exclusión. Luego, las políticas multiculturalistas no son la causa de la desconfianza y la falta de solidaridad, sino medidas que se han tomado a consecuencia de ellas, de manera que los grupos mayoritarios no teman o desprecien a las minorías y éstas últimas confíen en la sociedad ${ }^{30}$. El reconocimiento de todas las fuentes de dominación y opresión, incluidas por cierto las iniquidades étnico-culturales, no puede sino contribuir significativamente a la reducción de las injusticias y al fortalecimiento del Estado de Bienestar.

29. PAREKH, Bhikhu: Rethinking Multiculturalism, ob. cit. Págs. 366-367.

30. KYMLICKA, Will y BANTING, Keith (ed.): Multiculturalism and the Welfare State, ob. cit.

Revista de Ciencias Sociales - Número 67 (2015) - Universidad de Valparáíso - ISSN 0716-7725-Valparaíso, Chile 


\section{BIBLIOGRAFÍA}

BARRY, Brian: Culture and Equality: An Egalitarian Critique of Multiculturalism. Harvard University Press, Cambridge, 2002.

BAUMEISTER, Andrea: Liberalism and the 'Politics of Difference'. Edinburgh University Press, 2000.

CANEY, Simon: "Exceptions and Cultural Diversity". En: Kelly, Paul (ed.): Multiculturalism Reconsidered. Polity Press, Cambridge, 2002. Págs. 81-101.

DELANTY, Gerard: Community (2 ed.). Routledge, Abingdon, 2010.

FRASER, Nancy: "Rethinking Recognition". En: New Left Review, Mayo-Junio, No 3, 2000. Págs.107-120.

KYMLICKA, Will: Contemporary Political Philosophy: An Introduction ( $2^{\text {a }}$ ed). Oxford University Press, Oxford, 2002.

KYMLICKA, Will y BANTING, Keith (ed.): Multiculturalism and the Welfare State: Recognition and Redistribution in Contemporary

Democracies. Oxford University Press, Oxford, 2006.

MENDUS, Susan: "Choice, Chance and Multiculturalism”. En: Kelly, Paul (ed.): Multiculturalism Reconsidered. Polity Press, Cambridge, 2002. Págs. 31-44.

MILLER, David: "Multiculturalism and the Welfare State: Theoretical Reflections". En: Kymlicka, Will y Banting, Keith (ed.):

Multiculturalism and the Welfare State: Recognition and Redistribution in Contemporary Democracies. Oxford University Press, Oxford, 2006. Págs. 323-338.

MODOOD, Tariq: Multiculturalism. A Civic Idea. Polity, Cambridge, 2007.

PAREKH, Bhikhu (2002): "Barry and the Dangers of Liberalism". En: Kelly, Paul (ed.): Multiculturalism Reconsidered. Polity Press, Cambridge, 2002. Págs. 133-150.

—_ Rethinking Multiculturalism: Cultural Diversity and Political Theory (2 ed.). Palgrave MacMillan, Londres, 2006.

- (2008): A New Politics of Identity. Political principles for an Interdependent World. Palgrave MacMillan, Basingstoke, 2008.

SHAPIRO, Ian: "Democratic Justice, Multicultural Recognition". En: Kelly, Paul (ed.): Multiculturalism Reconsidered. Polity Press, Cambridge, 2002. Págs. 174-183.

Facultad de Derecho y Ciencias Sociales - Universidad de Valparaíso - Chile 
Squires, Judith: "Culture, Equality and Diversity". En: Kelly, Paul (ed.): Multiculturalism Reconsidered. Polity Press, Cambridge, 2002.

Págs. 114-132.

VILLAVICENCIO MIRANDA, Luis (2007): "La concepción política de la persona y las visiones constitutivas del bien”. En: Ideas y

Valores. Revista Colombiana de Filosofía, Universidad Nacional de Colombia, No 135, 2007. Págs. 29-49.

"Privatizando la diferencia: el liberalismo igualitario y el pluralismo cultural". En: Revista de Derecho (Valdivia), Volumen XXIII, Nº 1, 2010. Págs. 37-57.

YOUNG, Iris Marion: "A Multicultural Continuum: A Critique of Will Kymlicka's Ethnic-Nation Dichotomy”. En: Constellations, Volumen 4, No 1, 1997. Págs. 48-53.

_ La justicia y la política de la diferencia. Cátedra, Madrid, 2000. (Trad. Silvina Álvarez). 